OPEN ACCESS

Edited by:

Levi Wood,

Georgia Institute of Technology,

United States

Reviewed by:

Fabio Demontis,

St. Jude Children's Research Hospital, United States

Joo Young Huh,

Chonnam National University,

South Korea

*Correspondence: Constanza J. Cortes cjcortes@uab.edu

Specialty section:

This article was submitted to Dementia and Neurodegenerative

Diseases,

a section of the journa

Frontiers in Neurology

Received: 11 January 2021 Accepted: 25 May 2021

Published: 30 June 2021

Citation:

Gupta R, Khan R and Cortes CJ (2021) Forgot to Exercise? Exercise

Derived Circulating Myokines in Alzheimer's Disease: A Perspective.

Front. Neurol. 12:649452.

doi: 10.3389/fneur.2021.649452

\section{Forgot to Exercise? Exercise Derived Circulating Myokines in Alzheimer's Disease: A Perspective}

\author{
Rajesh Gupta ${ }^{1}$, Rizwan Khan ${ }^{1}$ and Constanza J. Cortes ${ }^{1,2,3,4 *}$ \\ ${ }^{1}$ Department of Cell, Developmental and Integrative Biology (CDIB), School of Medicine, University of Alabama at \\ Birmingham, Birmingham, AL, United States, ${ }^{2}$ Center for Neurodegeneration and Experimental Therapeutics (CNET), \\ University of Alabama at Birmingham, Birmingham, AL, United States, ${ }^{3}$ Center for Exercise Medicine, School of Medicine, \\ University of Alabama at Birmingham, Birmingham, AL, United States, ${ }^{4}$ UAB Nathan Shock Center for the Excellence in the \\ Study of Aging, University of Alabama at Birmingman, Birmingham, AL, United States
}

Regular exercise plays an essential role in maintaining healthy neurocognitive function and central nervous system (CNS) immuno-metabolism in the aging CNS. Physical activity decreases the risk of developing Alzheimer's Disease (AD), is associated with better AD prognosis, and positively affects cognitive function in AD patients. Skeletal muscle is an important secretory organ, communicating proteotoxic and metabolic stress to distant tissues, including the CNS, through the secretion of bioactive molecules collectively known as myokines. Skeletal muscle undergoes significant physical and metabolic remodeling during exercise, including alterations in myokine expression profiles. This suggests that changes in myokine and myometabolite secretion may underlie the well-documented benefits of exercise in AD. However, to date, very few studies have focused on specific alterations in skeletal muscle-originating secreted factors and their potential neuroprotective effects in $A D$. In this review, we discuss exercise therapy for $\mathrm{AD}$ prevention and intervention, and propose the use of circulating myokines as novel therapeutic tools for modifying AD progression.

Keywords: exercise, aging, myokines, exerkines, neuroprotection

\section{PATHOPHYSIOLOGY OF ALZHEIMER'S DISEASE}

Alzheimer's disease $(\mathrm{AD})$ is the most prevalent neurodegenerative disease affecting more than $10 \%$ of the human population over the age of 65 (1). AD is characterized by impaired executive function, language, and visual processing, eventually leading to dementia, memory loss and circadian and metabolic alterations (1). Pathologically these symptoms are associated with a loss of synapses, neurons, and an overall reduction of gray matter in vulnerable brain regions, including the hippocampus and the cortex. $\mathrm{AD}$ is also associated with two classical histopathological hallmarks: first, the accumulation of extracellular neuritic plaques, which are deposits of varied sizes of a small peptide known as amyloid- $\beta(A \beta)$, synthesized through sequential proteolytic cleavages of the amyloid- $\beta$ precursor protein (APP). Second, neurofibrillary tangles (NFTs) constituted of hyperphosphorylated microtubule-associated protein tau within affected neurons. These pathological hallmarks accumulate in affected brain areas, including the hippocampus and neocortical regions, and can be detected in the CNS decades before the onset of clinical symptoms 
(1). Currently there is no cure for $\mathrm{AD}$ and most available therapeutics focus on treating symptoms. (1). With estimates predicting around 12 million U.S. cases and 81 million worldwide by 2040 if preventive therapies are not found, the looming public health crisis stresses the urgency to develop novel interventions to prevent or delay the onset and progression of $\mathrm{AD}$.

\section{EXERCISE AND NEUROPROTECTION}

Regular exercise plays an essential role in maintaining healthy neurocognitive function and immuno-metabolism in the aging central nervous system (CNS), benefitting cognition and memory during healthy aging $(2,3)$ and reducing the risk of ageassociated neurodegenerative disease $(4,5)$. Indeed, multiple studies in rodent models have demonstrated that exercise, including forced treadmill and voluntary wheel running, powerfully stimulates adult hippocampal neurogenesis (4, 68 ) and increases brain derived neurotrophic factor (BDNF) levels in the aging hippocampus (7-9). Indeed, the CNS undergoes a progressive functional decline during aging, including increased neuroinflammation and dysfunction of proteostasis and mitochondrial systems (10-12), all of which are believed to contribute to the development of $\mathrm{AD}$ and can be modified through exercise interventions (Figure 1). This strongly implicates exercise as a highly relevant behavioral strategy for preventing age-related cognitive decline $(3,4,8)$. Although healthy aging and $\mathrm{AD}$ are two distinct multifactorial processes (1, 12), aging does remain the highest risk factor for the development of $\mathrm{AD}$ (3). This suggests that strategies that bolster CNS resilience during aging may also have important benefits in the prevention of and intervention against AD.

Moreover, physical activity decreases the risk of developing $\mathrm{AD}$ (13-15), is associated with better AD prognosis (16), and positively affects cognitive function in $\mathrm{AD}$ patients $(17,18)$. Indeed, individuals that participated in consistent physical activity in midlife reduced their risk for Alzheimer's disease by half (19), highlight the potent neuroprotective effects of exercise. Similar benefits have been reported in AD transgenic mice, where exercise rescues impaired neurogenesis, enhances synaptic plasticity, and attenuates $\mathrm{AD}$ associated-neuropathology (Figure 1) (20-25). However, the precise mechanisms responsible for this exercise-dependent rejuvenation of the aging and/or AD CNS remain largely unexplored.

Physical activity can include occupational, sports, conditioning, household, or other activities. Exercise is a subset of physical activity that is planned, structured, and repetitive, with the ultimate objective of improving or maintaining physical fitness (26). Endurance exercise training (running, dancing, cycling), leads to adaptations in both the cardiovascular and musculoskeletal system, supported by muscle hypertrophy and local increases in skeletal muscle mitochondrial biogenesis, capillary density, and oxidative capacity. Resistance exercise (strength training), on the other hand, mostly elicits changes in size and contractile properties of muscle, with direct adaptations on the neuromuscular system which support maximal motor unit synchronization and muscle activation. Since most of the
CNS benefits observed in human studies appear to occur after endurance training, suggesting differential biochemical and physiological responses to each exercise intervention (27), we will focus on the effects of this training modality in $\mathrm{AD}$ in this review.

\section{SKELETAL MUSCLE IS AN ENDOCRINE ORGAN SECRETING CNS-TARGETING "MYOKINES"}

Skeletal muscle has been proposed as a central regulator of organismal metabolism, communicating metabolic and proteostasis stress to distant tissues including brain via secretion of circulating "myokines" (28-30). In agreement with this, multiple epidemiological studies suggest that skeletal muscle aging is a risk factor for the development of age-associated disease $(28,31)$, including those of the CNS $(5,32)$. Skeletal muscle undergoes prominent remodeling during aging (3335 ), and exercise powerfully opposes these local deleterious effects, activating metabolic $(31,36)$ and proteostasis pathways to accommodate increased bioenergetic demands (37). Exercise also increases secretion of specific CNS-targeting myokines ("exercise induced myokines") into circulation, including BDNF (7), FNDC5/irisin (38), and cathepsin B (39), which very likely contribute to the exercise-associated benefits on cognition (39) (Figure 2). Indeed, shared circulation and young plasma injection experiments have suggested that circulating exerciseinduced myokines can promote functional rejuvenation of the aging neurogenic niche $(11,40)$. Consistent with this hypothesis, circulating blood factors in plasma from exercised aged mice are sufficient to transfer the effects of exercise on adult neurogenesis and cognition to sedentary aged mice (41) (Figure 2). Furthermore, conditioned media from activated muscle cells induces differentiation of adult neural stem cells in vitro (42), directly linking muscle originating secreted factors to the function of the aging CNS. Studies in Drosophila have also demonstrated a novel role for skeletal muscle in protecting the brain from age-related proteostasis collapse (43) and the role of nutrient-sensing myokines in regulating dopamine production in the CNS (44). However, to date, our knowledge of circulating myokines and their role in $\mathrm{AD}$ remains largely unexplored.

One consistent molecular biomarker of exercise in the brain is the induction of neurotrophins, particularly BDNF. BDNF promotes multiple aspects of CNS biology, including neuronal survival and differentiation, synaptogenesis, and synaptic plasticity. BDNF expression is robustly induced in the hippocampus with exercise (45), and active BDNF signaling is required for the exercise-associated benefits on memory and learning (46). However, the underlying mechanisms inducing BDNF activation in the hippocampus in response to exercise remain largely unknown. Recently, several exercise-associated myokines have arisen as novel regulators of BDNF activity during exercise (Figure 2). In this section, we discuss our current limited understanding of exercise-responsive skeletal muscle secreted factors and their effects on hallmarks of CNS function 

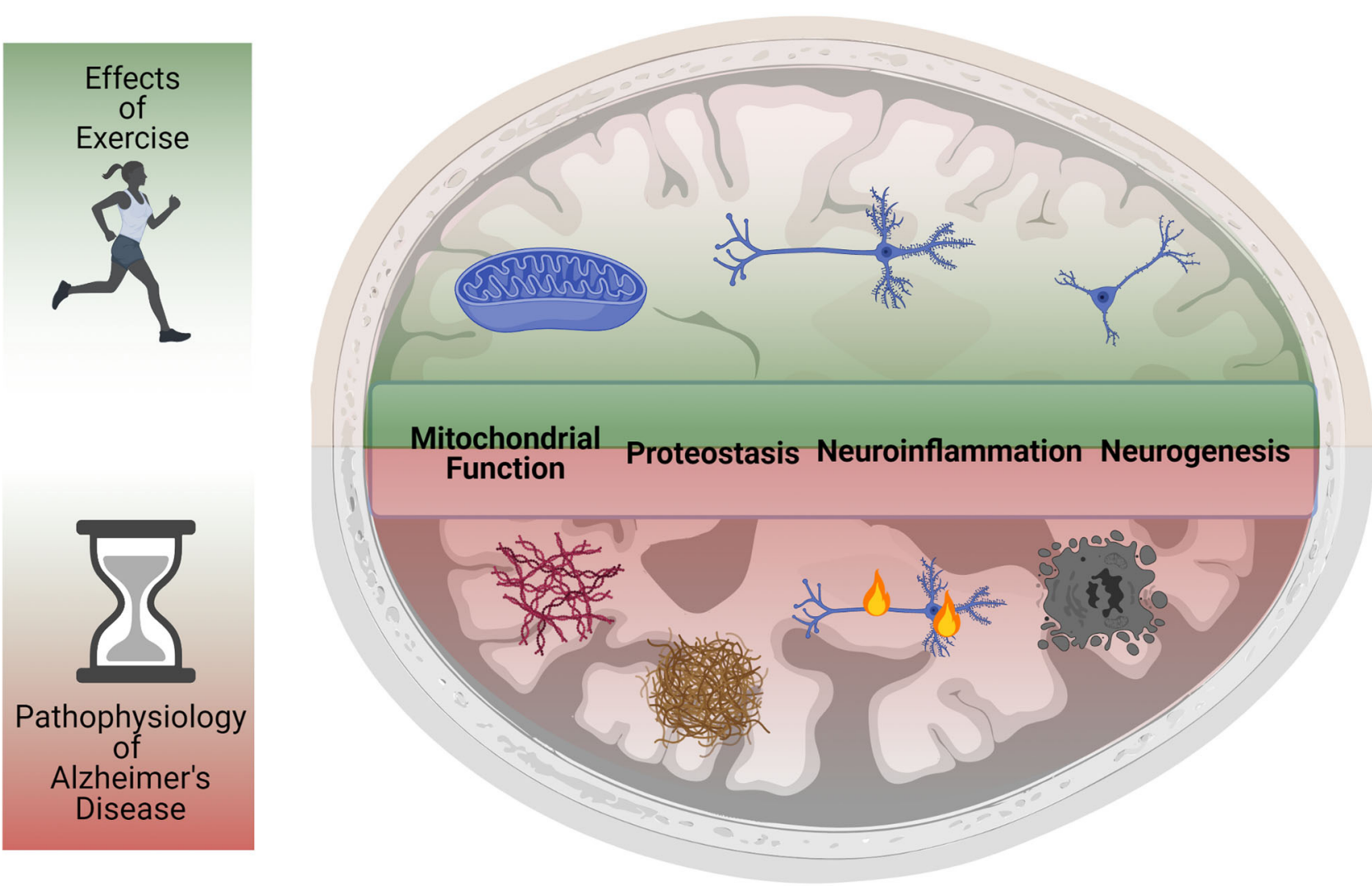

FIGURE 1 | Exercise reduces primary and secondary pathological hallmarks of Alzheimer's Disease. Studies in humans and animal models have shown that physical activity and exercise can have powerful protective effects in the CNS. Exercise reduces neuroinflammation, increases mitochondrial function, improves proteostasis, and rescues impaired neurogenesis. Animal model studies have also demonstrated that exercise reduces the levels of amyloid beta plaques and neurofibrillary tangles, preventing neurodegeneration and preserving cognitive function. The ability of exercise to improve most (if not all) of the pathological hallmarks of $A D$ suggests it may be a powerful intervention against the onset and progression of AD.

and/or $\mathrm{AD}$ pathologies, focusing on plaque deposition, tau hyperphosphorylation and neuroinflammation.

\section{FNDC5/Irisin}

FNDC5 is a glycosylated type I membrane protein, present in skeletal muscle and adipose tissue. During exercise, FNDC5 undergoes proteolytic cleavage and is released into circulation (47). This cleaved form of FNDC5, named "irisin," can be detected in both plasma and cerebrospinal fluid (CSF) (48), and its levels change depending on age, sex and underlying pathological conditions impacting skeletal muscle metabolism $(48,49)$. Furthermore, levels of circulating irisin increase in human and mouse serum post-exercise $(38,48,50)$ (Figure 2). Interestingly, circulating levels of FNDC5/irisin were reduced in the CSF and serum of a small cohort of $\mathrm{AD}$ patients (38). Exogenous delivery of FNDC5/irisin rescued synaptic plasticity and memory defects in a mouse model of $A \beta$-peptide hippocampal toxicity (38) and appears to reduce accumulation of hyperphosphorylated tau and increase levels of synaptic proteins in the hippocampus of human Tau transgenic mice (51). However, the mechanisms responsible for these effects remain currently unknown.
In addition to the neurotrophic effects of FNDC5/irisin, recent work has also suggested it may act as a novel potential regulator of neuroinflammation (52), another one of the central hallmarks of AD pathology (1). Peripheral delivery of recombinant irisin protein via intraperitoneal injection was sufficient to reduce levels of pro-neuroinflammatory markers GFAP, interleukin-1 $\beta$ (IL-1 $\beta$ ) and interleukin-6 (IL-6) in the CNS of a streptozotocininduced diabetic mouse model (52). Although whether similar anti-inflammatory effects for FNDC5/irisin occur in models of $\mathrm{AD}$ is currently unknown, the Brain Seq-Atlas suggests that the highest levels of expression ofIrisinand its putative receptors are found in astrocytes. This raises the possibility of FNDC5/irisin as a novel regulator of astrogliosis and pro-inflammatory signaling in the CNS, although this hypothesis remains yet to be tested.

It is currently unknown whether the peripheral (circulating) pool of FNDC5/irisin, which is highly responsive to exercise, contributes to the increased levels of FNDC5/irisin in the CNS after exercise. However, adenoviral vector delivery of FNDC5 to the mouse liver elevated circulating irisin levels, which in turn induced expression of BDNF in the hippocampus (50), suggesting that manipulation of peripheral FNDC5/irisin levels is sufficient to activate neurotrophic signaling in the CNS. 


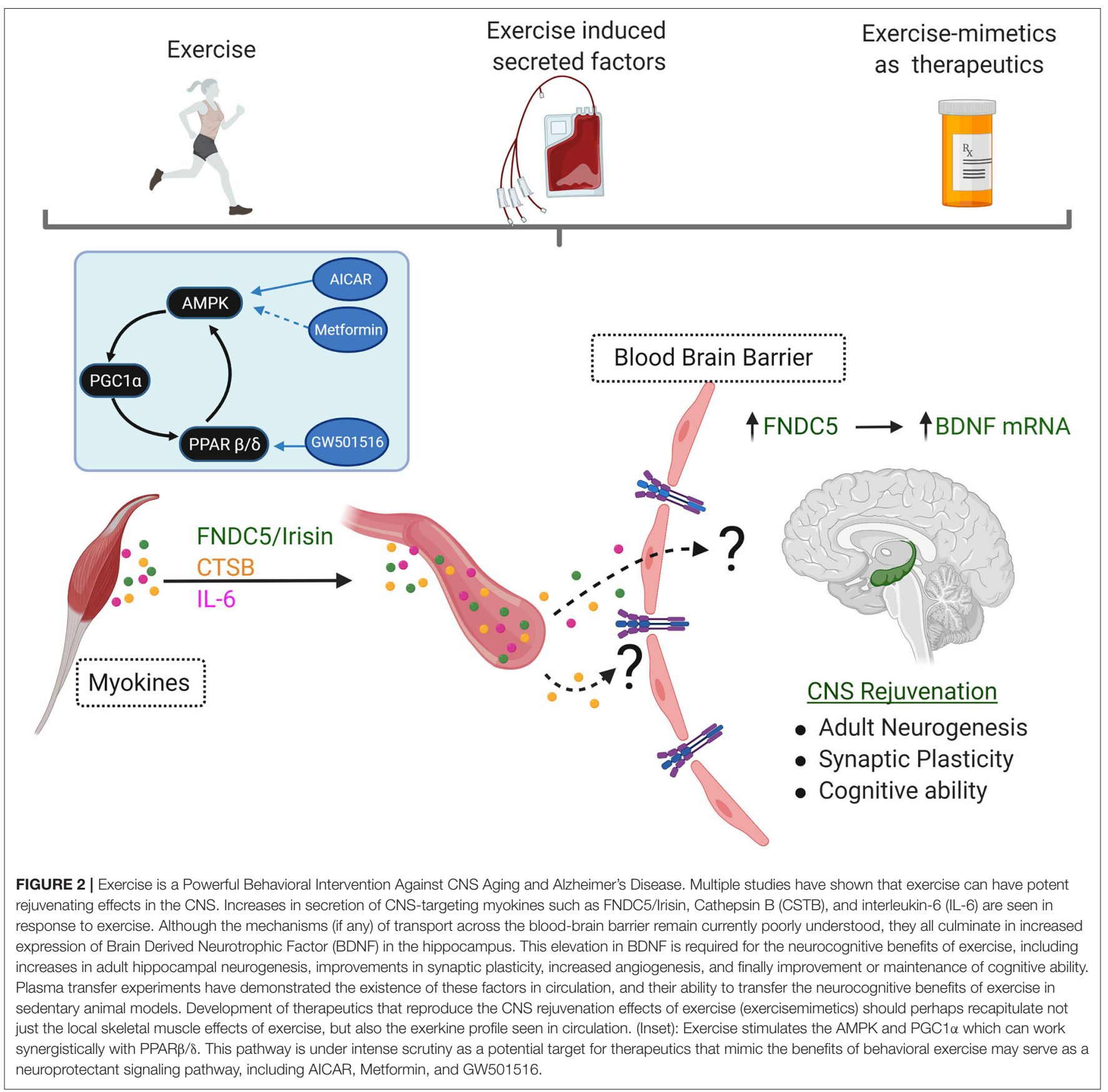

This highlights the potential of FNDC5/irisin as a novel, noninvasive therapeutic for $\mathrm{AD}$, which is currently an area of hot investigation.

\section{Cathepsin B}

Cathepsin B (CTSB), a lysosomal cysteine protease, is another muscle-originating circulating factor known to be upregulated in skeletal muscle, plasma, and the hippocampus after running in rodents, rhesus monkeys and humans (39) (Figure 2). Like FNDC5/irisin, administration of recombinant cathepsin B to adult hippocampal progenitor cells increases levels of BDNF and expression of doublecortin, a marker for neurogenesis (39). Long-term exercise training modulates peripheral levels CTSB and improves memory in middle-aged men, implicating CTSB as a direct mediator of the neurotrophic effects of exercise (53). In agreement with this, CTSB knock-out mice fail to enhance adult hippocampal neurogenesis and spatial memory function after running (39), suggesting that CTSB is required for the full manifestation of the neurocognitive benefits of exercise. Interestingly, CSTB can also directly degrade A $\beta$ assemblies (54) and lentiviral overexpression of CTSB reduces existing plaque deposits in hAPP mice (55). Cathepsin B also promotes the 
processing and secretion of mature-IL- $1 \beta$ through activation of microglia $(56,57)$ in a model of chronic pain. Similar antineuroinflammatory effects were detected in a systemic LPS exposure model leading to AD-like phenotypes in the CNS (58), although additional studies clarifying the role of CTSB in neuroinflammation in established AD models are needed.

Like FNDC5/irisin, the precise relationship between peripheral/circulating vs. central/hippocampal levels of CTSB remains currently poorly understood. However, it is interesting that whole-brain levels of CTSB are elevated in CTSB-KO mice after peripheral intravenous injection of recombinant CTSB (39), suggesting that at least some of the peripheral pool of CTSB is permeable through the blood-brain barrier. Cathepsin $B$ thus represents a potentially ideal exercise pharmaco-mimetic target: it is responsive to exercise, can activate hippocampal BDNF signaling and may possesses direct antiamyloidogenic and anti-neuroinflammation properties.

However, it is important to note that the role of CTSB in CNS function is controversial: as a lysosomal protease, increases in CTSB levels have also been associated with neuronal cell death in response to transient ischemia and traumatic brain injury (59). Furthermore, it appears that the specificity of CTSB to cleave APP is markedly reduced in the presence of the APP Swedish mutation, one of the causative genes for familial AD (60). Further studies probing the specific cleavage sites and activity, as well as any potential competition/cooperation between CTSB and other $\beta$-secretase-like proteases should help illuminate these apparent contradictory results.

\section{Interleukin-6}

Exercise increases local production and secretion of interleukin6 (IL-6) in skeletal muscle $(61,62)$, which is followed by a rapid decline after the end of the exercise period in healthy individuals $(61,63)$. Currently recognized as an exercise-inducible myokine, the biological output of IL-6 signaling is paradoxical. On one hand, chronically elevated levels of IL- 6 appear to be a signal for inflammation, are transduced via a cleaved, soluble ligand-bound IL-6 Receptor (a phenomenon known as "trans" IL-6 signaling) and are associated with most of the pathogenic action of IL6 in the CNS (64). Increased levels of IL-6 in the aged brain can be neurotoxic and affect cognitive and motivational systems (65). Altered levels of classical pro-inflammatory interleukins is a well-described feature of AD (66-68), with elevations of IL6 levels found in the blood and brain tissues of $\mathrm{AD}$ patients $(67,69)$. Moreover, the severity of dementia in cognitive disorders like Down syndrome and AD is associated with increased IL-6 levels (70). Further, activated microglia and astrocytes surround amyloid plaques in $\mathrm{AD}$, suggesting neurotoxic effects of cytokines (1). Interestingly, IL-6 is present in these senile plaques and could be detectable prior to amyloid plaque formation (1, 71 ), suggesting inflammatory cytokines may serve as primers for neuropathological changes as seen in development of $\mathrm{AD}$. Indeed, IL-6 can cross the blood-brain barrier, suggesting that chronic levels of peripheral inflammation may modulate central inflammatory processes and contribute to age-associated neurocognitive decline (ie. the "inflammaging" hypothesis) (12).
Conversely, transient elevations of IL-6, such as those seen during exercise, appear to play an anti-inflammatory role $(61,72)$ via "classical" IL-6 signaling, where the IL-6 ligand binds to a membrane-bound form of IL- 6 receptor. Indeed, some studies have shown that the acute increases in IL- 6 levels caused by exercise inhibit the activation of pro-inflammatory factor TNF $\alpha$ (73) and promote anti-inflammatory signaling (Figure 2) (74). Exercise also plays an important role modulating brain cytokine levels, altering the immune profile and improving cognitive performance and decreased amyloid deposition in the brain of Tg2576 Alzheimer's disease transgenic mice (75). Interestingly a recent a study showed that 16 weeks of exercise paradigm in AD patients increases plasma IL- 6 in the exercise group, compared to control group (76), suggesting similar exercise-associated muscle IL-6 responses in $\mathrm{AD}$ and healthy control groups. Although it is currently unclear whether IL-6 is necessary for the CNStargeting benefits of exercise, one interesting hypothesis is that transient IL-6 signaling originating from skeletal muscle may favor "classical" over "trans"-IL6 signaling, or perhaps be enriched for different populations of the ten currently identified members of the IL- 6 family. Given the underlying elevations in IL-6 levels in circulation in patients with $\mathrm{AD}$, understanding the contributions of acute vs. chronic IL-6 signaling, as well as identifying the downstream effects of exercise-associated vs. aging-associated responses in IL-6 secretion from skeletal muscle will be fundamental in the development of exercisemimicking therapeutics.

\section{EXERCISE-MIMICKING THERAPEUTIC INTERVENTIONS: THE AMPK-PGC- $1 \alpha-P P A R \beta / \Delta$ PATHWAY}

Exercise is an effective intervention against a wide variety of metabolic problems, age-related loss of function, and physiological complications. However, the benefits of behavioral exercise interventions remain inaccessible to many of the most at-risk populations, either due to economic status, immobility, or advancing disease. Although it is unlikely that a single medication or therapeutic target will achieve the plethora of benefits of exercise on brain and body physiology (77), identification of agents that mimic or potentiate the neurotrophic effects of endurance exercise are a highly attractive target for $\mathrm{AD}$ therapeutic development (Figures 1, 2). Multiple studies have focused on the metabolic networks and transcriptional activators involved in the beneficial roles of exercise in skeletal muscle although to date very little is known about their effects on myokine synthesis and secretion.

5' AMP-activated protein kinase (AMPK) is a master regulator of cellular metabolism, facilitating the energetic switch toward cellular catabolism in skeletal muscle during exercise. AMPK activation has both rapid effects induced via direct phosphorylation of downstream metabolic enzymes (metabolic reprogramming), and slower, more sustained effects achieved by transcriptional reprogramming through phosphorylation of PPAR $\gamma$ coactivator 1-alpha (PGC1 $\alpha$ ), a master regulator of mitochondrial biogenesis (77). Peroxisome proliferator-activated 
receptor (PPAR) PPAR $\beta / \delta$ is a PGC1 $\alpha$ co-factor with known effects on mitochondrial biogenesis, lipid metabolism and oxidative processes, and is also required for the transcriptional adaptations to exercise in skeletal muscle. The skeletal muscle AMPK-PGC-1 $\alpha$ pathway is one of the most examined signaling network in the field of exercise adaptations (77), yielding multiple pharmaceutical compounds and genetic interventions that mimic some of the local and CNS benefits of exercise.

For example, systemic administration of a well-known AMPK agonist, 5-Aminoimidazole-4-carboxamide 1- $\beta$-Dribofuranoside (AICAR) to male young C57/BL6 mice activated $\mathrm{BDNF}$ and neurogenesis in the dentate gyrus of the hippocampus to levels equivalent to those seen in untreated runners (78). Even in 2-year-old mice, longer AICAR treatment improves memory and motor coordination (79). Interestingly, these effects are likely to be due to peripheral activation of AICAR-sensitive pathways, given the low permeability of this compound through the bloodbrain barrier. In agreement with this, the AICAR-associated improvements in spatial memory are lost in muscle-specific AMPK $\alpha 2$ mutant mice (79), suggesting that activation of AMPK signaling may be an essential requirement for the CNS-targeting effects of exercise (Figure 2).

Metformin, another compound believed to activate AMPK signaling and with well-documented metabolic corrective effects, was recently found to act as a neuroprotective agent in a mouse model of $\mathrm{AD}$ (80), although these effects were only seen in females. Metformin can also act as a bonafide neuroprotectant, activating neurogenesis, increasing focal angiogenesis, and attenuating ischemia-induced brain injury in mice (81). Supported by lifespan-extension studies in rodents, metformin is currently being tested in human pre-clinical trials as a novel gero-protectant (Metformin in Longevity Study, MILES). Characterization of the biological fingerprint of aging in skeletal muscle before and after metformin treatment may yield important information of metformin-dependent transcriptional reprogramming associated with protection against age-associated disease, including any potential changes in exercise-induced myokines. Furthermore, long-term follow-up of study participants in the metformin arm of the trial may help to determine any potential protection against the development of AD.

PPAR $\delta$ acts synergistically with PGC1 $\alpha$ during exerciseassociated muscle remodeling. GW501516 is a well-characterized $\mathrm{PPAR} \beta / \delta$ agonist, promoting endurance-associated benefits on skeletal muscle (Figure 2) (77). In addition to multiple metabolism-associated benefits on skeletal muscle and liver of both genetic and induced mouse models of obesity, some evidence also suggests that GW501516 may have also some anti-inflammatory effects (82). Like AICAR, GW501516 has low blood-brain barrier permeability, implicating effects on the periphery as main drivers of the observed metabolic improvements. In agreement with this, important CNS benefits were seen after systemic GW501516 treatment in a mouse model of Huntington's Disease (83), directly implicating peripheral $\operatorname{PPAR} \beta / \delta$ as powerful novel neuroprotective pathway from the periphery into the CNS. Interestingly, direct infusion of GW501516 into the CNS may also have neuroprotective effects, activating neurogenesis and enhancing spatial memory in young female mice (84) and reducing both ischemia- and MPTPinduced neuronal toxicity (85). To our knowledge, no studies have examined changes in skeletal muscle-originating exercise induced myokines after GW501516 peripheral treatments but may represent a currently unexplored mechanism for the neuroprotective effects of GW501516 in models of neurodegenerative disease.

It is important to note that manipulation of individual members of the AMPK-PGC- $1 \alpha$ pathway sometimes has also failed to yield effects on the CNS. For example, musclespecific overexpression of $\mathrm{PGC1} \alpha$, a major regulator of the constitutively developed endurance muscle phenotype in rodents, was insufficient in preventing age-associated declines in hippocampal neurogenesis (86). Furthermore, the benefits of AICAR treatment on neurocognitive function in the CNS are transient, disappearing after 2 weeks of treatment. This is likely due to elevations in circulating and local pro-inflammatory cytokines with prolonged AICAR treatment (78). Because of this, understanding the long-term effects of these medications on neurocognitive function in humans-and separating these effects from existing co-morbidities or co-medications-will be essential, particularly for long-standing FDA approved compounds such as metformin.

Indeed, the question of exactly what cellular and biochemical remodeling pathways are activated via these pharmacological and/or genetic interventions remains: for example, although both exercise and GW501516 increase mitochondria fatty acid oxidation and fat metabolism in skeletal muscle, their downstream effects diverge. While exercise acts by promoting catabolism, glycolysis and gluconeogenesis in skeletal muscle, GW501516 treatment regulates branched chain amino acid and ketone body pathways instead (87). These studies suggest that exercise-mimetics that produce isolated changes in muscle consistent with those of exercise, independent of changes in exercise induced myokines profiles, may lead to unwanted counter-productive off target-effects (e.g., inflammation), failing to achieve sustainable neurotrophic effects on the CNS. On-going multi-site studies mapping the dynamic responses to exercise in metabolic tissues, including the brain, in response to different exercise modalities (27), will be essential to identify central pathways for targeted therapeutic developments of exercise-associated rejuvenation of the CNS.

It should be noted that dysregulation of AMPK signaling is prevalent in $\mathrm{AD}$, suggesting that further manipulation of this pathway may, at best, fail to achieve the observed benefits when used in combination of existing $\mathrm{AD}$-associated pathologies and at worst, exacerbate AD-associated symptoms. Further clarification of the specific nodes of AMPK-signaling altered in $\mathrm{AD}$ will facilitate therapeutic applications of AICAR and related compounds in AD. Finally, given the sex-specific metabolic dysfunction observed in some AD studies (88) and the sexdifferences reported in pre-clinical AMPK activator studies $(78,80)$, one additional potential area of investigation lies in the study of sex-differences in metabolic-targeting as an $\mathrm{AD}$ therapeutic intervention. 


\section{APOE STATUS AND EXERCISE}

Apolipoprotein E (APOE) status may modify the threshold for associations between physical exercise and dementia (89). Indeed, multiple lines of evidence suggest stronger protective effects of exercise in carriers of the APOE $\varepsilon 4$ allele $(19,90-92)$, one of the highest genetic risk factors for $\mathrm{AD}$, found in almost half of the late-onset $\mathrm{AD}$ cases (93). For example, a population based study of more than 1,600 older adults assessing the interaction between $A P O E$ status and exercise on dementia risk over a 5-year period suggests that physical exercise significantly moderated the relationship between genotype and dementia (94). In a posthoc analysis of 200 patients with mild $\mathrm{AD}$, patients who carried the APOE4 allele benefited more from the exercise intervention than non-carriers on cognitive, neuropsychiatric, and physical measures, suggesting that behavioral exercise interventions may also provide benefits after the onset of $\mathrm{AD}$ (95). Although these results have sometimes not been replicated (96-98) or flat out contradicted (99), we believe this reflects the complex relationship between exercise modalities, $\mathrm{AD}$ disease state and $A P O E$ status. Analysis of myokine profiles (before and after exercise) on APOE populations may yield novel information about skeletal muscle responses in the context of this highrisk gene.

\section{CURRENT CHALLENGES}

The variability of exercise paradigms used in human and animal model interventional trials (100), including high-intensity interval training (HIIT), resistance exercise, aerobic exercise, and multi-modal training, all associated with different intensities and durations, further complicates the identification of an "exercise prescription" for targeted therapeutic effects. Unification of duration and intensity of exercise-interventions in future clinical studies will be essential to allow comparisons across genotype, age, and disease progression. Indeed, human studies have demonstrated clear individual variations in post-exercise training metabolic adaptations (101), and individuals that already had cognitive impairments require higher levels of physical activity to achieve similar neurocognitive benefits (19). Inclusion of other factors including baseline fitness, dietary factors, training history, and between-session recovery during trials may also help explain these seemingly contradictory results (27). Finally, the historical underrepresentation of both women and nonCaucasian populations on exercise trials has prevented our understanding of the role of hormones and genetic diversity on exercise responses. On-going efforts to characterize tissue responses to these different exercise modalities in human populations and murine models (27) will yield key information for future behavioral exercise interventions with CNS benefits.

The benefits of physical activity and/or exercise are numerous and well-documented, although the precise mechanisms responsible for the observed improvements in cardiovascular, neurocognitive and well-being measurements remain poorly understood (Figure 1) (27). However, even if exercise interventions, be it behavioral or pharmacological, do not alter the known pathological hallmarks of $\mathrm{AD}$, their use as adjuvants to correct downstream biological processes in combination with other therapeutic interventions targeting $\mathrm{AD}$ pathological findings is highly attractive from a clinical and financial standpoint. If exercise interventions could delay the progression of $\mathrm{AD}$ and impact nursing home placement and hospice care, for example, would lead to major benefits for patients, caretakers and medical personnel in the fight against $\mathrm{AD}$.

\section{CONCLUSION}

The number of identified circulating factors produced and released from exercising skeletal muscle continues to grow. Indeed, the skeletal muscle secretome contains both free and membrane-bound bioactive factors, including proteins, metabolites, and micro-RNAs. Furthermore, the effects of exercise on the muscle secretome profile are only barely beginning to be understood. The construction of an atlas of exercise-responsive myokine or myokine-associated nodes through the on-going Molecular Transducers of Physical Activity Consortium (MoTRPAC) studies (27), as well as specific modifications to these networks with aging or disease (including $\mathrm{AD}$ ), will inform potential therapeutic interventions that reproduce the neuroprotective effects of exercise. Locally, exercise pharmaco-mimetics should reproduce the local effects on skeletal muscle, including re-programming the established muscle fiber specification toward an overt endurance phenotype, and activation of mitochondrial proteostasis and functional pathways. They should reproduce similar myokine profiles in circulation and ideally be orally active. Improving CNS resilience during aging, either through behavioral, pharmacological, or combinatorial approaches, should thus be a high-priority target for our field. Importantly, this will require integrated efforts of multiple scientific communities, including neuroscientists, clinicians, and exercise physiologists.

\section{AUTHOR CONTRIBUTIONS}

RG, RK, and CC worked together in the development of this manuscript and wrote the initial drafts of the manuscript. RG and RK performed literature searches and created the associated figures, with direct input and supervision from CC. CC edited and compiled the final version.

\section{FUNDING}

This work was funded by the National Institute of Aging (7RF1AG057264 and 7R03AG063215). 


\section{REFERENCES}

1. Masters CL, Bateman R, Blennow K, Rowe CC, Sperling RA, Cummings JL. Alzheimer's disease. Nat Rev Dis Primers. (2015) 1:15056. doi: $10.1038 /$ nrdp. 2015.56

2. Moon HY, Praag HV. Physical activity and brain plasticity. J Exerc Nutr Biochem. (2019) 23:23-25. doi: 10.20463/jenb.2019.0027

3. Kramer AF, Colcombe S. Fitness effects on the cognitive function of older adults: a meta-analytic study-revisited. Perspect Psychol Sci. (2018) 13:2137. doi: $10.1177 / 1745691617707316$

4. Choi SH, Bylykbashi E, Chatila ZK, Lee SW, Pulli B, Clemenson $\mathrm{GD}$, et al. Combined adult neurogenesis and BDNF mimic exercise effects on cognition in an Alzheimer's mouse model. Science. (2018) 361:eaab8821. doi: 10.1126/science.aan8821

5. Kim J, Choi KH, Cho SG, Kang SR, Yoo SW, Kwon SY, et al. Association of muscle and visceral adipose tissues with the probability of Alzheimer's disease in healthy subjects. Sci Rep. (2019) 9:949. doi: 10.1038/s41598-018-37244-9

6. van Praag H, Kempermann G, Gage FH. Running increases cell proliferation and neurogenesis in the adult mouse dentate gyrus. Nat Neurosci. (1999) 2:266-70. doi: 10.1038/6368

7. Marlatt MW, Potter MC, Lucassen PJ, van Praag H. Running throughout middle-age improves memory function, hippocampal neurogenesis, and BDNF levels in female C57BL/6J mice. Dev Neurobiol. (2012) 72:94352. doi: 10.1002/dneu.22009

8. van Praag H, Shubert T, Zhao C, Gage FH. Exercise enhances learning and hippocampal neurogenesis in aged mice. J Neurosci. (2005) 25:86805. doi: 10.1523/JNEUROSCI.1731-05.2005

9. Kronenberg G, Bick-Sander A, Bunk E, Wolf C, Ehninger D, Kempermann G. Physical exercise prevents age-related decline in precursor cell activity in the mouse dentate gyrus. Neurobiol Aging. (2006) 27:150513. doi: 10.1016/j.neurobiolaging.2005.09.016

10. Kempermann G, Song H, Gage FH. Neurogenesis in the adult hippocampus. Cold Spring Harb Perspect Biol. (2015) 7:a018812. doi: 10.1101/cshperspect.a018812

11. Villeda SA, Luo J, Mosher KI, Zou B, Britschgi M, Bieri G, et al. The ageing systemic milieu negatively regulates neurogenesis and cognitive function. Nature. (2011) 477:90-4. doi: 10.1038/nature10357

12. Lopez-Otin C, Blasco MA, Partridge L, Serrano M, Kroemer G. The hallmarks of aging. Cell. (2013) 153:1194-217. doi: 10.1016/j.cell.2013.05.039

13. Paillard T, Rolland Y, de Souto Barreto P. Protective effects of physical exercise in alzheimer's disease and parkinson's disease: a narrative review. J Clin Neurol. (2015) 11:212-9. doi: 10.3988/jcn.2015.11.3.212

14. Friedland RP, Fritsch T, Smyth KA, Koss E, Lerner AJ, Chen CH, et al. Patients with Alzheimer's disease have reduced activities in midlife compared with healthy control-group members. Proc Natl Acad Sci USA. (2001) 98:3440-5. doi: 10.1073/pnas.061002998

15. Lautenschlager NT, Cox KL, Flicker L, Foster JK, van Bockxmeer FM, Xiao J, et al. Effect of physical activity on cognitive function in older adults at risk for Alzheimer disease: a randomized trial. JAMA. (2008) 300:102737. doi: 10.1001/jama.300.9.1027

16. Scarmeas N, Luchsinger JA, Brickman AM, Cosentino S, Schupf N, XinTang M, et al. Physical activity and Alzheimer disease course. Am J Geriatr Psychiatry. (2011) 19:471-81. doi: 10.1097/JGP.0b013e3181eb00a9

17. Phillips C, Baktir MA, Das D, Lin B, Salehi A. The link between physical activity and cognitive dysfunction in alzheimer disease. Phys Ther. (2015) 95:1046-60. doi: 10.2522/ptj.20140212

18. Yang SY, Shan CL, Qing H, Wang W, Zhu Y, Yin MM, et al. The effects of aerobic exercise on cognitive function of alzheimer's disease patients. CNS Neurol Disord Drug Targets. (2015) 14:12927. doi: 10.2174/1871527315666151111123319

19. Rovio S, Kåreholt I, Helkala EL, Viitanen M, Winblad B, Tuomilehto $\mathrm{J}$, et al. Leisure-time physical activity at midlife and the risk of dementia and Alzheimer's disease. Lancet Neurol. (2005) 4:705-11. doi: 10.1016/S1474-4422(05)70198-8

20. Rodriguez JJ, Noristani HN, Olabarria M, Fletcher J, Somerville TD, Yeh CY, et al. Voluntary running and environmental enrichment restores impaired hippocampal neurogenesis in a triple transgenic mouse model of Alzheimer's disease. Curr Alzheimer Res. (2011) 8:70717. doi: 10.2174/156720511797633214

21. Hu YS, Xu P, Pigino G, Brady ST, Larson J, Lazarov O, et al. Complex environment experience rescues impaired neurogenesis. FASEB J. (2010) 24:1667-81. doi: 10.1096/fj.09-136945

22. Huttenrauch M, Brauss A, Kurdakova A, Borgers H, Klinker F, Liebetanz D, et al. Physical activity delays hippocampal neurodegeneration and rescues memory deficits in an Alzheimer disease mouse model. Transl Psychiatry. (2016) 6:e800. doi: 10.1038/tp.2016.65

23. Chao F, Jiang L, Zhang Y, Zhou C, Zhang L, Tang J, et al. Stereological investigation of the effects of treadmill running exercise on the hippocampal neurons in middle-aged APP/PS1 transgenic mice. J Alzheimers Dis. (2018) 63:689-703. doi: 10.3233/JAD-171017

24. Belarbi K, Burnouf S, Fernandez-Gomez FJ, Laurent C, Lestavel S, Figeac $M$, et al. Beneficial effects of exercise in a transgenic mouse model of Alzheimer's disease-like Tau pathology. Neurobiol Dis. (2011) 43:48694. doi: 10.1016/j.nbd.2011.04.022

25. Ohia-Nwoko O, Montazari S, Lau YS, Eriksen JL. Long-term treadmill exercise attenuates tau pathology in P301S tau transgenic mice. Mol Neurodegener. (2014) 9:54. doi: 10.1186/1750-1326-9-54

26. Caspersen CJ, Powell KE, Christenson GM. Physical activity, exercise, and physical fitness: definitions and distinctions for health-related research. Public Health Rep. (1985) 100:126-31.

27. Sanford JA, Nogiec CD, Lindholm ME, Adkins JN, Amar D, Dasari S, et al. Molecular transducers of physical activity, molecular transducers of physical activity consortium (MoTrPAC): mapping the dynamic responses to exercise. Cell. (2020) 181:1464-74. doi: 10.1016/j.cell.2020.06.004

28. Rai M, Demontis F. Systemic nutrient and stress signaling via myokines and myometabolites. Annu Rev Physiol. (2016) 78:85-107. doi: 10.1146/annurev-physiol-021115-105305

29. Agudelo LZ, Femenía T, Orhan F, Porsmyr-Palmertz M, Goiny M, Martinez-Redondo V, et al. Skeletal muscle PGC- $1 \alpha 1$ modulates kynurenine metabolism and mediates resilience to stress-induced depression. Cell. (2014) 159:33-45. doi: 10.1016/j.cell.2014.07.051

30. Katsimpardi L, Litterman NK, Schein PA, Miller CM, Loffredo FS, Wojtkiewicz GR, et al. Vascular and neurogenic rejuvenation of the aging mouse brain by young systemic factors. Science. (2014) 344:6304. doi: $10.1126 /$ science. 1251141

31. Cartee GD, Hepple RT, Bamman MM, Zierath JR. Exercise promotes healthy aging of skeletal muscle. Cell metabolism. (2016) 23:103447. doi: 10.1016/j.cmet.2016.05.007

32. Cortes CJ, Ling SC, Guo LT, Hung G, Tsunemi T, Ly L, et al. Muscle expression of mutant androgen receptor accounts for systemic and motor neuron disease phenotypes in spinal and bulbar muscular atrophy. Neuron. (2014) 82:295-307. doi: 10.1016/j.neuron.2014.03.001

33. Ubaida-Mohien C, Lyashkov A, Gonzalez-Freire M, Tharakan R, Shardell M, Moaddel R, et al. Discovery proteomics in aging human skeletal muscle finds change in spliceosome, immunity, proteostasis and mitochondria. Elife. (2019) 8:e49874. doi: 10.7554/eLife.49874

34. Uchitomi R, Hatazawa Y, Senoo N, Yoshioka K, Fujita M, Shimizu T, et al. Metabolomic analysis of skeletal muscle in aged mice. Sci Rep. (2019) 9:10425. doi: 10.1038/s41598-019-46929-8

35. Welle S, Brooks AI, Delehanty JM, Needler N, Thornton CA. Gene expression profile of aging in human muscle. Physiol Genomics. (2003) 14:149-59. doi: 10.1152/physiolgenomics.00049.2003

36. Mansueto G, Armani A, Viscomi C, D’Orsi L, De Cegli R, Polishchuk EV, et al. Transcription factor EB controls metabolic flexibility during exercise. Cell Metab. (2017) 25:182-96. doi: 10.1016/j.cmet.2016.11.003

37. Pillon NJ, Gabriel BM, Dollet L, Smith JAB, Sardon Puig L, Botella J, et al. Transcriptomic profiling of skeletal muscle adaptations to exercise and inactivity. Nat Commun. (2020) 11:470. doi: 10.1038/s41467-019-13869-w

38. Lourenco MV, Frozza RL, de Freitas GB, Zhang H, Kincheski GC, Ribeiro FC, et al. Exercise-linked FNDC5/irisin rescues synaptic plasticity and memory defects in Alzheimer's models. Nat Med. (2019) 25:165175. doi: 10.1038/s41591-018-0275-4

39. Moon HY, Becke A, Berron D, Becker B, Sah N, Benoni G, et al. RunningInduced systemic cathepsin B secretion is associated with memory function. Cell Metab. (2016) 24:332-40. doi: 10.1016/j.cmet.2016.05.025 
40. Villeda SA, Plambeck KE, Middeldorp J, Castellano JM, Mosher KI, Luo J, et al. Young blood reverses age-related impairments in cognitive function and synaptic plasticity in mice. Nat Med. (2014) 20:659-63. doi: 10.1038/nm.3569

41. Horowitz AM, Fan X, Bieri G, Smith LK, Sanchez-Diaz CI, Schroer $\mathrm{AB}$, et al. Blood factors transfer beneficial effects of exercise on neurogenesis and cognition to the aged brain. Science. (2020) 369:16773. doi: $10.1126 /$ science.aaw2622

42. Moon HY, Javadi S, Stremlau M, Yoon KJ, Becker B, Kang $\mathrm{SU}$, et al. Conditioned media from AICAR-treated skeletal muscle cells increases neuronal differentiation of adult neural progenitor cells. Neuropharmacology. (2019) 145:12330. doi: 10.1016/j.neuropharm.2018.10.041

43. Demontis F, Perrimon N. FOXO/4E-BP signaling in Drosophila muscles regulates organism-wide proteostasis during aging. Cell. (2010) 143:81325. doi: 10.1016/j.cell.2010.10.007

44. Robles-Murguia M, Rao D, Finkelstein D, Xu B, Fan Y, Demontis F. Muscle-derived Dpp regulates feeding initiation via endocrine modulation of brain dopamine biosynthesis. Genes Dev. (2020) 34:37-52. doi: 10.1101/gad.329110.119

45. Park H, Poo MM. Neurotrophin regulation of neural circuit development and function. Nat Rev Neurosci. (2013) 14:7-23. doi: 10.1038/nrn3379

46. Vaynman S, Ying Z, Gomez-Pinilla F. Hippocampal BDNF mediates the efficacy of exercise on synaptic plasticity and cognition. Eur J Neurosci. (2004) 20:2580-90. doi: 10.1111/j.1460-9568.2004.03720.x

47. Bostrom P, Wu J, Jedrychowski MP, Korde A, Ye L, Lo JC, et al. A PGC1alpha-dependent myokine that drives brown-fat-like development of white fat and thermogenesis. Nature. (2012) 481:463-8. doi: 10.1038/nature10777

48. Fagundo AB, Jimenez-Murcia S, Giner-Bartolome C, Aguera Z, Sauchelli $S$, Pardo $M$, et al. Modulation of irisin and physical activity on executive functions in obesity and morbid obesity. Sci Rep. (2016) 6:30820. doi: 10.1038/srep30820

49. Chang JS, Kim TH, Nguyen TT, Park KS, Kim N, Kong ID. Circulating irisin levels as a predictive biomarker for sarcopenia: a cross-sectional communitybased study. Geriatr Gerontol Int. (2017) 17:2266-73. doi: 10.1111/ggi.13030

50. Wrann CD, White JP, Salogiannnis J, Laznik-Bogoslavski D, Wu J, Ma D, et al. Exercise induces hippocampal BDNF through a PGC-1 $\alpha$ /FNDC5 pathway. Cell Metab. (2013) 18:649-59. doi: 10.1016/j.cmet.2013.09.008

51. Bretland KA, Lin L, Bretland KM, Smith MA, Fleming SM, Dengler-Crish CM. Irisin treatment lowers levels of phosphorylated tau in the hippocampus of pre-symptomatic female but not male htau mice. Neuropathol Appl Neurobiol. (2021) 1-12. doi: 10.1111/nan.12711

52. Wang K, Song F, Xu K, Liu Z, Han S, Li F, et al. Irisin attenuates neuroinflammation and prevents the memory and cognitive deterioration in streptozotocin-induced diabetic mice. Mediators Inflamm. (2019) 2019:1567179. doi: 10.1155/2019/1567179

53. De la Rosa A, Solana E, Corpas R, Bartrés-Faz D, Pallàs M, Vina J, et al. Long-term exercise training improves memory in middle-aged men and modulates peripheral levels of BDNF and cathepsin B. Sci Rep. (2019) 9:3337. doi: 10.1038/s41598-019-40040-8

54. Sun B, Zhou Y, Halabisky B, Lo I, Cho SH, Mueller-Steiner S, et al. Cystatin $\mathrm{C}$-cathepsin $\mathrm{B}$ axis regulates amyloid beta levels and associated neuronal deficits in an animal model of Alzheimer's disease. Neuron. (2008) 60:24757. doi: 10.1016/j.neuron.2008.10.001

55. Mueller-Steiner S, Zhou Y, Arai H, Roberson ED, Sun B, Chen $\mathrm{J}$, et al. Antiamyloidogenic and neuroprotective functions of cathepsin B: implications for Alzheimer's disease. Neuron. (2006) 51:703-14. doi: 10.1016/j.neuron.2006.07.027

56. Sun L, Wu Z, Hayashi Y, Peters C, Tsuda $M$, Inoue $K$, et al. Microglial cathepsin $\mathrm{B}$ contributes to the initiation of peripheral inflammation-induced chronic pain. J Neurosci. (2012) 32:11330. doi: 10.1523/JNEUROSCI.0677-12.2012

57. Wu Z, Sun L, Hashioka S, Yu S, Schwab C, Okada R, et al. Differential pathways for interleukin- $1 \beta$ production activated by chromogranin A and amyloid $\beta$ in microglia. Neurobiol Aging. (2013) 34:271525. doi: 10.1016/j.neurobiolaging.2013.05.018

58. Wu Z, Ni J, Liu Y, Teeling JL, Takayama F, Collcutt A, et al. Cathepsin B plays a critical role in inducing Alzheimer's disease-like phenotypes following chronic systemic exposure to lipopolysaccharide from porphyromonas gingivalis in mice. Brain Behav Immun. (2017) 65:35061. doi: 10.1016/j.bbi.2017.06.002

59. Hook G, Jacobsen JS, Grabstein K, Kindy M, Hook V. Cathepsin B is a new drug target for traumatic brain injury therapeutics: evidence for e64d as a promising lead drug candidate. Front Neurol. (2015) 6:178. doi: 10.3389/fneur.2015.00178

60. Hook VY, Kindy M, Hook G. Inhibitors of cathepsin B improve memory and reduce beta-amyloid in transgenic Alzheimer disease mice expressing the wild-type, but not the Swedish mutant, beta-secretase site of the amyloid precursor protein. J Biol Chem. (2008) 283:774553. doi: 10.1074/jbc.M708362200

61. Pedersen BK. Exercise and cytokines. Immunol Cell Biol. (2000) 78:5325. doi: 10.1111/j.1440-1711.2000.t01-11-.x

62. Pedersen BK, Fischer CP. Physiological roles of muscle-derived interleukin-6 in response to exercise. Curr Opin Clin Nutr Metab Care. (2007) 10:26571. doi: 10.1097/MCO.0b013e3280ebb5b3

63. Petersen AMW, Pedersen BK. The anti-inflammatory effect of exercise. $J$ Appl Physiol. (2005) 98:1154-62. doi: 10.1152/japplphysiol.00164.2004

64. Campbell IL, Erta M, Lim SL, Frausto R, May U, Rose-John S, et al. Trans-signaling is a dominant mechanism for the pathogenic actions of interleukin-6 in the brain. J Neurosci. (2014) 34:250313. doi: 10.1523/JNEUROSCI.2830-13.2014

65. Papanicolaou DA, Wilder RL, Manolagas SC, Chrousos GP. The pathophysiologic roles of interleukin-6 in human disease. Ann Intern Med. (1998) 128:127-37. doi: 10.7326/0003-4819-128-2-19980115000009

66. Griffin WS, Stanley LC, Ling C, White L, MacLeod V, Perrot LJ, et al. Brain interleukin 1 and S-100 immunoreactivity are elevated in down syndrome and Alzheimer disease. Proc Natl Acad Sci USA. (1989) 86:7611. doi: 10.1073/pnas.86.19.7611

67. Licastro F, Pedrini S, Caputo L, Annoni G, Davis LJ, Ferri C, et al. Increased plasma levels of interleukin-1, interleukin- 6 and $\alpha$ 1-antichymotrypsin in patients with Alzheimer's disease: peripheral inflammation or signals from the brain? J Neuroimmunol. (2000) 103:97102. doi: 10.1016/S0165-5728(99)00226-X

68. Su F, Bai F, Zhang Z. Inflammatory cytokines and alzheimer's disease: a review from the perspective of genetic polymorphisms. Neurosci Bull. (2016) 32:469-80. doi: 10.1007/s12264-016-0055-4

69. Eriksson UK, Pedersen NL, Reynolds CA, Hong M-G, Prince JA, Gatz $\mathrm{M}$, et al. Associations of gene sequence variation and serum levels of C-reactive protein and interleukin-6 with alzheimer's disease and dementia. J Alzheimers Dis. (2011) 23:361-9. doi: 10.3233/JAD-2010-1 01671

70. Kalman J, Juhasz A, Laird G, Dickens P, Jardanhazy T, Rimanoczy A, et al. Serum interleukin-6 levels correlate with the severity of dementia in down syndrome and in Alzheimer's disease. Acta Neurol Scand. (1997) 96:236-40. doi: 10.1111/j.1600-0404.1997.tb00275.x

71. Bauer J, Strauss S, Schreiter-Gasser U, Ganter U, Schlegel P, Witt I, et al. Interleukin-6 and alpha-2-macroglobulin indicate an acutephase state in Alzheimer's disease cortices. FEBS Lett. (1991) 285:1114. doi: 10.1016/0014-5793(91)80737-N

72. Scheller J, Chalaris A, Schmidt-Arras D, Rose-John S. The pro- and antiinflammatory properties of the cytokine interleukin-6. Biochim Biophys Acta. (2011) 1813:878-888. doi: 10.1016/j.bbamcr.2011.01.034

73. Philippe M, Krusmann PJ, Mersa L, Eder EM, Gatterer H, Melmer A, et al. Acute effects of concentric and eccentric exercise on glucose metabolism and interleukin-6 concentration in healthy males. Biol Sport. (2016) 33:1538. doi: 10.5604/20831862.1198634

74. Steensberg A, Fischer CP, Keller C, Moller K, Pedersen BK. IL-6 enhances plasma IL-1ra, IL-10, and cortisol in humans. Am J Physiol Endocrinol Metab. (2003) 285:E433-7. doi: 10.1152/ajpendo.00074.2003

75. Parachikova A, Nichol KE, Cotman CW. Short-term exercise in aged Tg2576 mice alters neuroinflammation and improves cognition. Neurobiol Dis. (2008) 30:121-9. doi: 10.1016/j.nbd.2007.12.008

76. Jensen CS, Bahl JM, Østergaard LB, Høgh P, Wermuth L, Heslegrave A, Zetterberg $\mathrm{H}$, et al. Exercise as a potential modulator of inflammation in patients with Alzheimer's disease measured in cerebrospinal fluid and plasma. Exp Gerontol. (2019) 121:91-8. doi: 10.1016/j.exger.2019.04.003 
77. Narkar VA, Downes M, Yu RT, Embler E, Wang YX, Banayo E, et al. AMPK and PPARdelta agonists are exercise mimetics. Cell. (2008) 134:40515. doi: 10.1016/j.cell.2008.06.051

78. Guerrieri D, van Praag H. Exercise-mimetic AICAR transiently benefits brain function. Oncotarget. (2015) 6:18293-313. doi: 10.18632/oncotarget.4715

79. Kobilo T, Guerrieri D, Zhang Y, Collica SC, Becker KG, van Praag H. AMPK agonist AICAR improves cognition and motor coordination in young and aged mice. Learn Mem. (2014) 21:119-26. doi: 10.1101/lm.033332.113

80. DiTacchio KA, Heinemann SF, Dziewczapolski G. Metformin treatment alters memory function in a mouse model of Alzheimer's disease. $J$ Alzheimers Dis. (2015) 44:43-8. doi: 10.3233/JAD-141332

81. Liu Y, Tang G, Zhang Z, Wang Y, Yang GY. Metformin promotes focal angiogenesis and neurogenesis in mice following middle cerebral artery occlusion. Neurosci Lett. (2014) 579:46-51. doi: 10.1016/j.neulet.2014.07.006

82. Salvado L, Barroso E, Gomez-Foix AM, Palomer X, Michalik L, Wahli W, et al. PPARbeta/delta prevents endoplasmic reticulum stressassociated inflammation and insulin resistance in skeletal muscle cells through an AMPK-dependent mechanism. Diabetologia. (2014) 57:212635. doi: 10.1007/s00125-014-3331-8

83. Dickey AS, Sanchez DN, Arreola M, Sampat KR, Fan W, Arbez N, et al. PPARdelta activation by bexarotene promotes neuroprotection by restoring bioenergetic and quality control homeostasis. Sci Transl Med. (2017) 9:eaal2332. doi: 10.1126/scitranslmed.aal2332

84. Kobilo T, Yuan C, van Praag H. Endurance factors improve hippocampal neurogenesis and spatial memory in mice. Learn Mem. (2011) 18:1037. doi: $10.1101 / \mathrm{lm} .2001611$

85. Iwashita A, Muramatsu Y, Yamazaki T, Muramoto M, Kita Y, Yamazaki S, et al. Neuroprotective efficacy of the peroxisome proliferator-activated receptor delta-selective agonists in vitro and in vivo. J Pharmacol Exp Ther. (2007) 320:1087-96. doi: 10.1124/jpet.106.115758

86. Karlsson L, Gonzalez-Alvarado MN, Motalleb R, Blomgren K, Borjesson M, Kuhn HG. Constitutive PGC-1alpha overexpression in skeletal muscle does not protect from age-dependent decline in neurogenesis. Sci Rep. (2019) 9:12320. doi: 10.1038/s41598-019-48795-w

87. Chen W, Gao R, Xie X, Zheng Z, Li H, Li S, et al. A metabolomic study of the PPARdelta agonist GW501516 for enhancing running endurance in kunming mice. Sci Rep. (2015) 5:9884. doi: 10.1038/srep09884

88. Ferretti MT, Iulita MF, Cavedo E, Chiesa PA, Schumacher Dimech A, Santuccione Chadha A, et al. Sex differences in Alzheimer disease the gateway to precision medicine. Nat Rev Neurol. (2018) 14:45769. doi: 10.1038/s41582-018-0032-9

89. Low LF, Yap MH, Brodaty H. Will testing for apolipoprotein E assist in tailoring dementia risk reduction? A review. Neurosci Biobehav Rev. (2010) 34:408-37. doi: 10.1016/j.neubiorev.2009.08.007

90. Deeny SP, Poeppel D, Zimmerman JB, Roth SM, Brandauer J, Witkowski S, et al. Exercise APOE, and working memory: MEG and behavioral evidence for benefit of exercise in epsilon4 carriers. Biol Psychol. (2008) 78:17987. doi: 10.1016/j.biopsycho.2008.02.007

91. Etnier JL, Caselli RJ, Reiman EM, Alexander GE, Sibley BA, Tessier D, et al. Cognitive performance in older women relative to ApoE-epsilon 4 genotype and aerobic fitness. Med Sci Sports Exerc. (2007) 39:199207. doi: 10.1249/01.mss.0000239399.85955.5e
92. Schuit AJ, Feskens EJ, Launer LJ, Kromhout D. Physical activity and cognitive decline, the role of the apolipoprotein e4 allele. Med Sci Sports Exerc. (2001) 33:772-7. doi: 10.1097/00005768-200105000-00015

93. Saunders AM, Schmader K, Breitner JC, Benson MD, Brown WT, Goldfarb L, et al. Apolipoprotein E epsilon 4 allele distributions in late-onset Alzheimer's disease and in other amyloid-forming diseases. Lancet. (1993) 342:7101. doi: 10.1016/0140-6736(93)91709-U

94. Fenesi B, Fang H, Kovacevic A, Oremus M, Raina P, Heisz JJ. Physical exercise moderates the\&nbsp;relationship of apolipoprotein E (APOE) genotype and dementia risk: a population-based study. J Alzheimers Dis. (2017) 56:297-303. doi: 10.3233/JAD-1 60424

95. Jensen CS, Simonsen AH, Siersma V, Beyer N, Frederiksen KS, Gottrup $\mathrm{H}$, et al. Patients with Alzheimer's disease who carry the APOE epsilon4 allele benefit more from physical exercise. Alzheimers Dement. (2019) 5:99106. doi: 10.1016/j.trci.2019.02.007

96. Lindsay J, Laurin D, Verreault R, Hébert R, Helliwell B, Hill GB, et al. Risk factors for Alzheimer's disease: a prospective analysis from the Canadian study of health and aging. Am J Epidemiol. (2002) 156:445-53. doi: 10.1093/aje/k wf074

97. Sabia S, Kivimaki M, Kumari M, Shipley MJ, Singh-Manoux A. Effect of apolipoprotein E epsilon4 on the association between health behaviors and cognitive function in late midlife. Mol Neurodegener. (2010) 5:23. doi: 10.1186/1750-1326-5-23

98. Taaffe DR, Irie F, Masaki KH, Abbott RD, Petrovitch H, Ross GW, et al. Physical activity, physical function, and incident dementia in elderly men: the honolulu-asia aging study. J Gerontol A Biol Sci Med Sci. (2008) 63:529-35. doi: 10.1093/gerona/63.5.529

99. Podewils LJ, Guallar E, Kuller LH, Fried LP, Lopez OL, Carlson $\mathrm{M}$, et al. Physical activity, APOE genotype, and dementia risk: findings from the cardiovascular health cognition study. Am J Epidemiol. (2005) 161:639-51. doi: 10.1093/aje/k wi092

100. Lam FM, Huang MZ, Liao LR, Chung RC, Kwok TC, Pang MY. Physical exercise improves strength, balance, mobility, and endurance in people with cognitive impairment and dementia: a systematic review. J Physiother. (2018) 64:4-15. doi: 10.1016/j.jphys.2017.12.001

101. Pickering C, Kiely J. Do non-responders to exercise exist-and if so, what should we do about them? Sports Med. (2019) 49:1-7. doi: 10.1007/s40279-018-01041-1

Conflict of Interest: The authors declare that the research was conducted in the absence of any commercial or financial relationships that could be construed as a potential conflict of interest.

Copyright (C) 2021 Gupta, Khan and Cortes. This is an open-access article distributed under the terms of the Creative Commons Attribution License (CC BY). The use, distribution or reproduction in other forums is permitted, provided the original author(s) and the copyright owner(s) are credited and that the original publication in this journal is cited, in accordance with accepted academic practice. No use, distribution or reproduction is permitted which does not comply with these terms. 